\title{
Chairmen's introduction
}

In March 2005 a unique meeting was held in Milan, Italy, which brought together international experts from three specialities - gastroenterology, cardiology and rheumatology. The aim of this meeting was to provide insight and guidance for clinicians across the globe regarding the balance of risks and benefits when prescribing non-steroidal anti-inflammatory drug (NSAID) treatment, particularly in patients at risk of gastrointestinal (GI) damage or those with cardiovascular (CV) risk factors.

Current management of a patient who requires NSAID therapy, such as those with rheumatoid arthritis, osteoarthritis or ankylosing spondylitis, is far from simple, in particular with regard to the choice of an effective, low risk therapeutic agent.

In September 2004, the pharmaceutical manufacturer Merck voluntarily withdrew rofecoxib, a cyclooxygenase-2 (COX-2) selective inhibitor, from the market. This decision was made after a chemoprevention study revealed that after 18 months of therapy, subjects receiving rofecoxib had roughly twice the rate of myocardial infarction and stroke when compared with the rate seen in placebo controls. ${ }^{1}$ On 07 April 2005, the US Food and Drug Administration requested that Pfizer remove valdecoxib from the market after concluding its benefits did not outweigh its comprehensive safety profile. ${ }^{2}$ COX-2 selective inhibitors, which until these recent events represented around one-third of all NSAID prescriptions in the USA, were originally developed to reduce the risk of GI adverse events commonly seen with NSAIDs, while still providing comparable symptom relief.

The withdrawal of two commonly prescribed COX-2 inhibitors used by millions of patients worldwide has prompted a re-evaluation by regulatory agencies of the safety of the whole class of COX-2 inhibitors. Prescribing clinicians are now weighing up the known risks of COX-2 inhibitors with the benefits of pain relief in considering whether this class of agents is appropriate for their patients who need anti-inflammatory therapy, especially those with underlying $\mathrm{CV}$ risk.
The discussions and recommendations made at this meeting, and summarized here, are intended to help and guide physicians in clinical practice when prescribing NSAIDs, including COX-2 inhibitors, for their patients, many of whom may also be taking low-dose aspirin for prophylaxis against cardiovascular events.

The views of the expert panel of rheumatologists, cardiologists and gastroenterologists from Europe, the USA and Asia were each considered in turn, taking into account existing international treatment guidelines, GI and CV risk definitions, and recent recommendations. The whole panel then debated these overlapping viewpoints in the light of the available evidence about COX-2 inhibitors, and developed recommendations for patient treatment with NSAIDs, dependent on their level of GI and/or CV risk. Overall it was concluded that in many patients traditional, non-selective NSAIDs may be used as an alternative to COX-2 inhibitors, however for those patients at risk of GI damage or those also taking aspirin, a gastroprotective agent, such as a proton-pump inhibitor, should also be prescribed.

We would like to thank the entire faculty involved in this meeting for their active participation and valuable input into the discussions and recommendations.

J. M. SCHEIMAN University of Michigan Medical Center, Ann Arbor, MI, USA

B. CRYer

University of Texas Southwestern Medical School, Dallas, TX, USA

\section{References}

1 Merck Announces Voluntary Worldwide Withdrawal of VIOXX $^{\circledR}$ [press release]. NJ: Whitehouse Station, September 30, 2004. Available at: http://www.vioxx.com/rofecoxib/vioxx/ consumer/index.jsp.

2 FDA. FDA [press release]. FDA Announces Important Changes and Additional Warnings for COX-2 Selective and Non-selective Non-steroidal Anti-inflammatory Drugs (NSAIDs), April 2005. http://www.fda.gov/cder/drug/advisory/COX2.htm. 